\title{
Les pratiques pédagogiques des enseignant-es universitaires tunisien-nes par les TIC
}

Adel Ben Taziri, Université Virtuelle de Tunis

Abdeljalil Akkari, Université de Genève

DOI : 10.51186/journals/ed.2020.10-2.e348

\section{Résumé}

Cet article présente les résultats de l'enquête quantitative menée auprès d'un échantillon d'enseignant-es de l'université tunisienne. Son objectif est de de comprendre la façon dont les enseignant-es vivent l'intégration des Technologies de l'Information et de la Communication (TIC) dans leurs pratiques professionnelles. Les résultats de cette enquête nous permettront également de vérifier si les stratégies institutionnelles concordent avec la perception des enseignant-es impliqué-es au sein de leurs établissements en termes de développement des TIC. Le modèle de Raby nous a permis de proposer un portrait actualisé de l'expertise des enseignant-es universitaires tunisien-nes en matière des pratiques pédagogiques par les TIC et d'identifier leurs compétences technologiques actuelles.

Mots-clés : pratiques pédagogiques, TIC, enseignants, université, Tunisie

\begin{abstract}
This article presents the results of a quantitative survey conducted among a sample of teachers at Tunisian universities. Its objective is to understand how teachers experience the integration of information and communication technologies (ICT) into their professional practices. The results of the survey will also allow us to determine whether the universities' institutional strategies align with the perceptions of teachers involved in ICT development in their schools. Using Raby's model, we provide an updated picture of Tunisian university teachers' expertise in ICT-based pedagogical practices and identify their current technological skills.
\end{abstract}

Keywords: pedagogical practices, ICT, teachers, university, Tunisia

\section{INTRODUCTION}

Comment L'intégration pédagogique des TIC est définie comme " une utilisation habituelle et régulière des TIC en classe par les élèves et les enseignants, dans un contexte d'apprentissage actif, réel et significatif, pour soutenir et améliorer l'apprentissage et l'enseignement » (Raby, 
2004, p. 23). Elle implique des modifications des pratiques des enseignant-es et touche profondément à leurs représentations de l'apprentissage, à leurs modalités de collaboration et d'évaluation, et à leur rapport au savoir. Cette intégration peut bouleverser les modes d'enseignement-apprentissage ainsi que le milieu de travail.

Dans les politiques d'introduction des technologies dans le secteur de l'éducation, deux objectifs sont souvent cités : le premier vise la démocratisation de l'accès aux TIC pour lutter contre la fracture numérique ; le second vise l'amélioration de la qualité des pratiques d'enseignement et d'apprentissage. D'autres auteur-es ont pu montrer que l'intégration pédagogique des TIC ne se fait pas sans difficulté (Karsenti, Savoie-Zajc \& Larose, 2001).

Nous nous intéressons dans cet article aux pratiques des enseignant-es universitaires tunisiennes par les TIC. En effet, l'intégration intensive des TIC dans l'enseignement supérieur tunisien date depuis 2002 avec la création de l'Université Virtuelle de Tunis (UVT). Nous avons repéré, dans plusieurs travaux de recherche (Allouche \& Belcadhi, 2016 ; Houissa, 2009 ; Jelmam, 2012 ; Kaddachi, 2017 ; Kalai, 2018 ; Zghidi, 2010), un nombre important de projets et d'initiatives d'intégration des TIC qui ont été réalisés dans le contexte universitaire tunisien, mais peu ont fait l'objet d'enquêtes et d'analyses documentées. Nous soulignons également que la documentation collectée traite d'une façon superficielle les pratiques et les usages des enseignant-es par les TIC. Les études et les publications scientifiques collectées sont restées limitées à quelques articles qui ne permettent ni de comprendre la dynamique de l'intégration des TIC dans l'enseignement supérieur, ni de faire une étude comparative entre les différentes universités concernant cette intégration. Toutefois, l'engagement des enseignant-es dans des projets TIC mérite d'être étudié, sans oublier que l'absence de littérature rend le travail de recherche encore plus complexe. Ainsi, notre objectif dans cet article est de proposer un portrait actualisé de l'expertise des enseignant-es universitaires tunisien-nes en matière d'usage des TIC et de comprendre la façon dont ces enseignant-es vivent l'intégration des technologies dans leurs pratiques professionnelles. Pour atteindre cet objectif, nous avons réalisé une enquête par questionnaire pour recueillir des données quantifiables concernant les usages des enseignant-es universitaires tunisien-nes par les TIC. La collecte des données a eu lieu pendant l'année académique 2015-2016 par la distribution d'un questionnaire à 907 enseignant-es de cinq établissements universitaires tunisiens.

La revue de la littérature exposée dans la prochaine section vise à mettre en évidence les enjeux et le potentiel de l'intégration pédagogique des TIC dans l'enseignement supérieur tunisien et nous nous intéressons essentiellement aux travaux de recherches sur les pratiques et les usages des TIC des enseignant-es tunisien-nes. 


\section{REVUE DE LITTÉRATURE}

Com Le faible nombre de chercheurs et chercheuses et leurs contributions scientifiques en Tunisie dans le domaine de l'intégration pédagogique des TIC a été relevé par l'enquête de Trouche, et al. (2014). En Tunisie, uniquement 42 répondants ont participé à cette enquête et ont déclaré que leurs recherches se situent dans le secteur de l'intégration pédagogique des TIC. La discipline la plus représentée est l'informatique avec 12 répondants, puis 9 en sciences de l'information et de la communication, 5 enseignant-es en sciences de l'éducation, et 3 en didactiques. Quant aux sujets de recherche, 6 répondants ont manifesté de l'intérêt pour I'enseignement à distance, 5 pour une didactique, 4 pour les recherches dans le champ de l'intégration pédagogique des TICE et 3 pour les environnements informatiques pour l'apprentissage humain (EIAH).

Dans ce que suit, nous présentons quelques travaux de recherches tunisiennes en étroite relation avec le sujet de cet article, c'est à dire, l'intégration des TIC dans l'enseignement supérieur tunisien.

Allouche et Belcadhi (2016) se sont intéressés à l'évaluation de l'enseignement transversal proposé par I'UVT. Les auteur-es ont montré que l'enseignement transversal de l'entrepreneuriat et du cours " projet professionnel personnalisé ", accompagné de l'utilisation des ressources pédagogiques numériques et d'autres actions de l'UVT (formations, partage d'expériences, réseautage), pourrait être un outil judicieux pour le perfectionnement à l'enseignement au profit des enseignant-es universitaires expérimenté-es. II peut également être proposé au profit des enseignant-es universitaires moins expérimenté-es à condition de leur offrir un accompagnement par les enseignant-es plus expérimenté-es.

Un autre travail de Allouche (2016) a donné un aperçu de la formation des enseignant-es tunisien-nes à l'intégration pédagogique des TIC. La principale conclusion de ce travail est l'existence, dans la cadre de la formation des enseignant-es en Tunisie, d'acquis importants et indéniables au niveau des acteurs et actrices, des programmes, des résultats, etc., et ce, au niveau de la formation initiale et de la formation continue.

Kalai (2018) a mené une enquête auprès d'enseignant-es et d'étudiant-e-es d'institutions du Kef sur les facteurs qui expliquent le non-usage des TIC ou la réticence à leur utilisation. Les principaux obstacles mentionnés par les participants sont relatifs à la politique et à la stratégie de mise en œuvre, à l'infrastructure et à la formation des enseignant-es.

Une autre étude, menée par Kaddachi (2017) en 2014 auprès de 221 enseignant-es universitaires, démontre que l'intégration des TIC dans la pédagogie universitaire tunisienne reste encore dans une phase relativement débutante. 
Quant à Benabid (2017), il a mené une étude sur l'évolution de l'utilisation de la plateforme Moodle chez un groupe d'étudiant-es inscrits à l'Institut supérieur des études technologiques de Kébili dans le cadre d'une formation hybride diplômante destinée aux adultes. L'étude, menée sur trois ans (2013-2016), est basée sur l'analyse des traces numériques fournies par la plateforme et les résultats d'entretiens semi-directifs réalisés avec les apprenant-es.

Khezami (2016), dans sa thèse, a cherché à comprendre le comportement autorégulé des apprenant-es tunisiens dans le cadre des formations en ligne de I'UVT. Cette recherche a abouti au résultat suivant :

Le dispositif UVT est basé sur les technologies numériques. De ce fait, le comportement autorégulé des apprenant-es oscille, selon le degré de contrôle pédagogique qu'exerce le dispositif UVT sur leur apprentissage, entre adaptation aux conditions formelles et création de conditions informelles contournant ainsi le dispositif de l'institution. (Khezami, 2016, p. 357)

Nous avons repéré également la thèse de Tabei $(2009,2010)$ qui s'est focalisé sur l'intégration des TIC dans le champ universitaire tunisien et spécifiquement dans le réseau des Instituts Supérieurs des Études Technologiques. Elle s'est concentrée sur les usages effectifs des apprenant-es et des enseignant-es dans le cadre du contexte d'enseignement à distance.

Nous étions attentifs aussi aux résultats des travaux publiés par Zghidi (2010) qui s'est intéressée aux transformations structurelles et pédagogiques qui caractérisent le domaine de l'enseignement, de la formation et de l'apprentissage inhérentes aux technologies de l'information et de la communication. Ses travaux s'inscrivent dans l'optique de l'évaluation des stratégies éducatives basées sur les dispositifs de l'enseignement à distance.

Nous avons également repéré des articles scientifiques qui portent sur les pratiques et les usages des enseignant-es et des étudiant-es par les TIC. Nous citons les travaux de Houissa (2009) et Jelmam (2012) qui ont présenté des retours d'expérience sur la mise en place d'un dispositif d'apprentissage collaboratif hybride dans le cadre de la préparation au Certificat en Informatique et Internet (C2i) au sein des leurs institutions. Ces études ont évalué l'écart entre les compétences numériques attendues et celles atteintes dans le cadre de ce cours intitulé C2i.

En somme, en tenant compte de tous ces travaux, nous synthétisons que les études sur les pratiques et les usages des enseignant-es par les TIC en enseignement supérieur ne fournissent pas une idée claire et détaillée sur les dispositifs développés et les pratiques des enseignant-es avec les technologies. 


\section{MÉTHODOLOGIE}

Plusieurs travaux ont tenté d'évaluer le degré d'intégration des TIC dans l'enseignement (Coen \& Schumacher, 2006 ; Moersch, 1995 ; Raby, 2004). En effet, dans ces travaux l'« intégration des TIC » n'est pas considérée comme un état de fait, mais comme un processus. Ces auteurs soulignent que ce processus se déroule en traversant une série d'étapes allant de la nonutilisation à une utilisation exemplaire.

Plusieurs des modèles ont été développés pour tracer ces étapes que traversent habituellement les enseignant-es lors de l'intégration des TIC à leur enseignement. Nous citons celui de Moersch (1995), de Coen et Shumacher (2006), et celui de l'UNESCO (2008). Nous nous sommes appuyés essentiellement sur le modèle de synthèse de Raby (2004) pour décrire la situation actuelle de l'utilisation des TIC par les enseignant-es universitaires tunisien-es. Ce modèle permet de tracer les étapes que traversent habituellement les enseignant-es lors de l'intégration des TIC à leur enseignement. Ces derniers illustrent un processus menant de la non-utilisation à l'utilisation exemplaire des TIC, se divise en quatre stades : la " sensibilisation ", l'« utilisation personnelle ", l'« utilisation professionnelle " et l'« utilisation pédagogique "

Rappelons que ce texte a pour objectif de tracer un portrait actualisé de l'expertise des enseignant-es universitaires tunisien-nes en matière d'usage des TIC et de comprendre la façon dont ces enseignant-es vivent l'intégration des technologies dans leurs pratiques professionnelles. Dans cette perspective, nous avons interrogé les enseignant-es sur les différents aspects de leur usage des TIC, depuis le stade de l'utilisation personnelle, passant par celui de l'utilisation professionnelle, jusqu'au stade de l'utilisation pédagogique de nature exploratoire, d'infusion ou d'appropriation. Pour ce faire, nous avons d'abord cherché à repérer à quel stade se positionnent ces enseignant-es lorsqu'on les interroge sur leurs pratiques en TIC. En effet, nous vérifions les compétences et utilisations des outils numériques des enseignant-es tels que: les médias numériques et outils de communication traditionnels (courriel, PowerPoint, PDF), les outils de communications sociaux (blogues, chats, forums et réseaux sociaux), les systèmes d'évaluation électronique en ligne, les médias audio-vidéo et tutoriels (YouTube, podcasts...), les outils interactifs (Wikis, visioconférence, outils en ligne tels que Google Drive, jeux éducatifs...), l'utilisation des plateformes d'enseignement en ligne, etc.

Nous nous sommes intéressés particulièrement au stade de l'utilisation pédagogique. Ce stade d'utilisation des TIC se subdivise en 5 étapes successives:

- L'étape de la motivation de l'enseignant-e à utiliser les TIC, que ce soit par curiosité, parce qu'il exprime un besoin de varier les occasions de son apprentissage, ou par une obligation provenant de la direction, par exemple.

- L'étape de la familiarisation est un premier pas se caractérisant par une valeur éducative limitée. Elle se réalise par appropriation technique basique et par utilisation simple des TIC. 
- L'étape de l'exploration amène l'enseignant-e à faire l'expérience de matériel informatisé soutenant son enseignement. Les apprenant-es y sont engagés dans des activités visant l'acquisition et l'application de connaissances.

- L'étape de l'infusion implique une utilisation beaucoup plus régulière des TIC, mais encore ponctuelle en termes de transmission et construction des connaissances par l'enseignant-e. Elle permet néanmoins le développement de compétences disciplinaires.

- L'étape de l'appropriation est caractérisée par une plus forte régularité dans l'utilisation des $\mathrm{TIC}$, et ce pour la construction et la transmission des connaissances permettant le développement de compétences disciplinaires et transversales.

\section{1. Échantillonnage et sélection des cas}

Les acteurs/trices du milieu universitaire concerné-es par cette étude sont les enseignant-es des cinq établissements universitaires sélectionnés. La sélection s'est basée sur des critères tels que la situation géographique, le nombre d'étudiant-es, le nombre d'enseignant-es et les champs disciplinaires des diplômes délivrés et ce dans l'objectif de prendre en compte toutes les situations d'intégration pédagogique des TIC dans l'enseignement supérieur tunisien. Ainsi, de nombreux filtres ont été utilisés pour aboutir à la sélection d'un nombre restreint de cinq cas. D'abord, une prospection a été faite auprès des responsables officiels des institutions universitaires où les TIC semblent intégrées. Une lettre de présentation du projet et des fiches de pré-identification ont été adressées aux responsables des établissements. Un questionnaire exploratoire en ligne a été ensuite envoyé aux responsables. La collecte de données nous a permis de classer hiérarchiquement les établissements en fonction des réponses de leurs responsables. Fondée sur l'élimination des établissements dont les réponses sont au-dessous du niveau 3 du modèle de Graham, et al. (2013), cette classification a eu lieu en deux étapes successives : une présélection des institutions dotées d'une stratégie institutionnelle ; et parmi ces dernières, la sélection des institutions pionnières en matière d'offre de formation en ligne. Enfin, nous avons retenu les cinq établissements universitaires suivant pour notre étude :

- La Faculté des Sciences Économiques et de Gestion de Sfax (FSEGS)

- L'Institut Supérieur des Études Technologiques de la Charguia (ISET-Charguia)

- L'Institut Supérieur d'Informatique et de Télécommunication (ISITCom)

- L'École Supérieure des Sciences Infirmières de Tunis (ISSIT)

- La Faculté des Sciences de Bizerte (FSB)

\subsection{Le questionnaire}

Le questionnaire, en annexe à cet article, a été construit et adopté en 2015. Par la suite, nous avons procédé à une pré-enquête pour nous assurer de la bonne compréhension des questions. Après des réajustements et des suppressions de questions pour donner suite aux différents tests que nous avons menés, notre questionnaire définitif comporte 46 questions réparties sur sept parties. La première s'intéresse au profil des répondants. La seconde partie permet d'identifier la façon avec laquelle les répondants ont été formés à l'intégration des TIC. La troisième partie met en avant les structures et les supports favorisant l'intégration des TIC 
dans le cadre universitaire. La quatrième partie est consacrée à leurs usages pédagogiques des TIC. Réservée aux enseignant-es qui ont proposé des cours intégrés via les plateformes de l'UVT, la cinquième partie du questionnaire met en lumière les motivations, les pratiques pédagogiques à distance et les modifications occasionnées par ces dernières. La sixième partie interroge ces derniers sur leurs usages des TIC. Enfin, la septième partie présente les avantages et les obstacles perçus de l'intégration des TIC dans l'enseignement.

Toutes les questions sont des questions fermées ou à choix multiples. L'ensemble des rubriques de ce questionnaire nous permet d'avoir une idée du profil de l'enseignant-e, de son rapport avec les TIC et du niveau d'intégration des TIC dans l'établissement où il exerce.

\subsection{Recueil et analyse de données}

Le questionnaire a été administré en ligne et ce du 11 février au 24 mars 2016. 314 enseignants-chercheurs et enseignantes-chercheuses ont répondu à notre questionnaire en ligne. $64 \%$ sont des femmes, $69 \%$ sont âgés de moins de quarante ans. Plus de la moitié a une expérience d'enseignement de six à quinze ans (58\%). Nous avons sélectionné 314 réponses complètes. Le Tableau 1 ci-dessous présente la répartition des répondant-es par établissement.

\section{Tableau 1. Répartition des répondant-es}

\begin{tabular}{|c|c|c|c|c|c|c|c|}
\hline \multicolumn{2}{|c|}{ Établissement } & FSEGS & ISET-Charguia & ISITCom & FSB & ISSIT & Total \\
\hline \multicolumn{2}{|c|}{ Ville -région } & Sfax-Sud & Tunis & Sousse-Centre & Bizerte - Nord & Tunis & \\
\hline \multicolumn{2}{|c|}{$\begin{array}{c}\text { Nombre total des enseignant- } \\
\text { es par établissement }\end{array}$} & 298 & 118 & 107 & 359 & 25 & 907 \\
\hline \multicolumn{2}{|c|}{ Nombre de répondants } & 97 & 92 & 42 & 69 & 14 & 314 \\
\hline \multicolumn{2}{|c|}{ Pourcentage des répondants } & $32,55 \%$ & $77,97 \%$ & $39,25 \%$ & $19,22 \%$ & $56,00 \%$ & $34,62 \%$ \\
\hline \multirow[b]{2}{*}{ Genre } & Femme & 65 & 60 & 18 & 49 & 10 & 199 \\
\hline & Homme & 32 & 32 & 24 & 25 & 4 & 115 \\
\hline \multirow{6}{*}{ Discipline } & Autre & 9 & 18 & 7 & 18 & 0 & 45 \\
\hline & $\begin{array}{l}\text { Lettres et } \\
\text { Sciences } \\
\text { Humaines }\end{array}$ & 6 & 2 & 0 & 12 & 0 & 20 \\
\hline & $\begin{array}{c}\text { Sciences } \\
\text { Fondamentales }\end{array}$ & 4 & 2 & 5 & 4 & 0 & 13 \\
\hline & $\begin{array}{c}\text { Sciences } \\
\text { économiques }\end{array}$ & 21 & 21 & 5 & 5 & 0 & 53 \\
\hline & $\begin{array}{l}\text { Sciences } \\
\text { Médicales }\end{array}$ & 0 & 0 & 1 & 0 & 10 & 18 \\
\hline & Informatique & 57 & 48 & 24 & 30 & 2 & 163 \\
\hline \multirow{2}{*}{$\begin{array}{l}\text { Collaboration } \\
\text { avec I'UVT }\end{array}$} & Non & 34 & 27 & 4 & 16 & 3 & 84 \\
\hline & Oui & 36 & 65 & 38 & 53 & 11 & 230 \\
\hline
\end{tabular}


Pour analyser les données recueillies, nous avons effectué un tri à plat du questionnaire. Nous avons ensuite élaboré des tableaux de contingence, en croisant à chaque fois les variables supposées explicatives et la variable à expliquer. Des tests de khi deux ont permis de préciser la significativité des liaisons observées.

\section{RÉSULTATS}

Ce paragraphe présente les résultats de l'enquête quantitative menée auprès d'un échantillon d'enseignant-es dans cinq institutions universitaires tunisiennes. Son objectif est de dresser un état des lieux des pratiques privées et publiques et des besoins des enseignant-es en TIC. Les résultats de cette enquête nous permettront également de vérifier si les stratégies institutionnelles concordent avec l'avis des enseignant-es impliqué-es au sein de leurs établissements dans le développement des TIC.

\subsection{La formation des enseignant-es à l'intégration pédagogique des TIC}

La formation des enseignant-es à l'intégration des TIC en pédagogie est évidemment une condition fondamentale pour améliorer la qualité des enseignements. La modalité analysée dans cette section concerne le statut des répondants en ce qui concerne la formation à I'intégration des TIC : 45\% des enseignant-es disent avoir une formation en TIC. En effet, les enseignant-es questionné-es pensent que ce type de formation permet à l'enseignant-e de modifier sa manière d'enseigner (91\%) en mettant en place de nouvelles activités d'apprentissage (89\%). Neuf enseignant-es sur 10 pensent que la formation leur a permis d'utiliser les outils de la plateforme et de devenir plus autonome et efficace dans leur utilisation de ces technologies.

\subsection{Les structures et les supports favorisant l'intégration des TIC dans les institutions universitaires}

Cette section cherche à identifier et à explorer les facteurs institutionnels qui influencent l'appropriation des TIC.

Nous avons pu vérifier, en premier lieu, si les enseignant-es disposent d'un ordinateur personnel. Les résultats montrent que $98 \%$ des enseignant-es sont équipé-es d'un ordinateur portable, 80 \% accèdent à Internet depuis leur domicile et 77\% disposent de smartphones avec accès à Internet.

Nous avons demandé, ensuite, aux enseignant-es de préciser les équipements disponibles dans leurs établissements. Nous remarquons que dans les cinq établissements, les vidéoprojecteurs (98\%) et les salles informatiques (65\%) sont disponibles et faciles à réserver. Cependant, les classes mobiles et les tablettes pour étudiant-es, qui constituent des TIC modernes, sont presque inexistantes dans tous ces établissements. 
L'un des principaux constats que nous avons pu faire au cours de nos enquêtes est la présence dans les établissements d'enseignement supérieur d'une ou de plusieurs salle(s) informatique(s) destinées à la formation en ligne. Malheureusement, dans la plupart des cas, ces salles ne sont pas en libre accès.

Nous avons également demandé aux enseignant-es d'évaluer la qualité des équipements informatiques dans leurs établissements. Pour 68\% des répondant-Es, les équipements disponibles et le réseau de leur établissement sont d'une qualité moyenne ou mauvaise. II faut dire aussi que pour des raisons principalement technologiques, les enseignant-es ont des difficultés de se connecter à Internet dans les salles de cours. La faiblesse des débits de connexion que nous avons pu observer dans la plupart des établissements dans lesquels nous avons enquêté, est une source de démotivation pour beaucoup d'entre eux. L'opinion des enseignant-es sur la qualité du réseau internet de leur établissement n'est majoritairement positive dans aucun établissement.

\subsection{Les usages des TIC}

En s'appuyant sur le modèle de Raby (2004), nous avons cherché à repérer à quel stade se positionnent ces enseignant-es lorsqu'on les interroge sur leurs pratiques avec les TIC.

a) Utilisation personnelle

Dans l'ensemble, I'utilisation personnelle des TIC par les enseignant-es tunisien-es est satisfaisante. En effet, neuf enseignant-es sur dix déclarent utiliser l'ordinateur dans le cadre de leurs cours, pour leur détente ou leur loisir, les ressources en ligne pour apprendre, communiquer avec des amis via des réseaux sociaux, tout en respectant la vie privée et les droits d'auteur-es. En somme, la quasi-totalité des enseignant-es répondant à cette enquête ont des pratiques privées par les TIC.

b) Utilisation professionnelle

La plupart des enseignant-es ayant suivi une formation à l'intégration des TIC utilisent un logiciel de présentation assistée par ordinateur de type PowerPoint (88\% des enseignant-es formés), utilisent Internet au cours des séances d'enseignement (69\%) et numérisent leurs supports de cours ( $91 \%$ des formés). Seuls 45\% des enseignant-es formé-es ont mis en ligne leurs cours via une plateforme d'enseignement à distance.

Les résultats de l'enquête montrent que l'utilisation des TIC pour partager des cours en ligne reste insuffisamment développée : seuls $44 \%$ des enseignant-es affirment que leurs étudiantes retrouvent leur cours sur une plateforme d'enseignement distance. 
Nous avons aussi choisi d'analyser l'utilisation professionnelle en mettant l'accent sur l'usage d'internet pendant les séances de cours. Dans leur écrasante majorité, les enseignant-es qui ont participé à notre enquête affirment utiliser Internet pendant les séances de cours, c'est le cas de 60\% des enseignant-es. Ce qui montre bien l'importance grandissante que prend cet outil d'information et de communication dans le milieu universitaire.

Le modèle théorique de Raby (2004) admet que l'utilisation personnelle des TIC dans l'enseignement est un antécédent à leur utilisation professionnelle. Afin de vérifier ce présupposé, nous avons analysé conjointement les différentes utilisations personnelles des TIC par les enseignant-es qui se sont confirmé-es utilisateurs d'Internet pendant leur cours.

Les résultats indiquent une forte association positive entre l'utilisation personnelle et l'utilisation professionnelle des TIC, dans la mesure où la grande majorité des utilisateurs d'Internet pendant les cours se sont révélés des « grands » utilisateurs et "grandes » utilisatrices personnel-les des TIC.

\section{c) L'utilisation pédagogique}

Nous nous intéressons aux trois stades de l'utilisation pédagogique des TIC à savoir : l'exploration, l'infusion et l'appropriation.

\section{1) L'exploration pédagogique des TIC}

Seuls 18\% des enseignant-es interrogé-es, affirment ne pas utiliser de logiciels de présentation pendant leurs cours. L'écrasante majorité des enquêté-es, qui répondait affirmativement à cette question avait cité particulièrement le logiciel PowerPoint.

Comme nous pouvions déjà le soupçonner, les enseignant-es les plus jeunes dans la profession (moins de quinze ans d'expérience) sont celles et ceux qui font un plus grand usage des logiciels de présentation.

S'agissant de la numérisation de cours et en tenant compte du pourcentage des enseignantes utilisant des logiciels de présentation assistée par ordinateur (PréAO), nous remarquons que le taux des affirmations des enseignant-es ayant numérisé leurs cours s'élève à $87 \%$.

La numérisation des cours est une réalité dans les cinq institutions sélectionnées et par tous les enseignant-es (toutes disciplines confondues et tous les grades). Uniquement $13 \%$ des enseignant-es interrogé-es affirment ne pas mettre leur cours en format numérique. Ce taux élevé de réponses positives pourrait s'expliquer comme découlant de la conclusion de certains ateliers de formation de l'UVT. Ces ateliers proposent en effet la mise en ligne d'un cours. Les enseignant-es apprennent ainsi à déposer sur une plateforme une petite partie de leur enseignement classique, après l'avoir structurée pour une mise en ligne. 
Malgré des difficultés persistantes, $86 \%$ des enseignant-es disent exploiter des outils de communication en ligne pour le suivi des étudiant-es. L'outil le plus utilisé est le mail (78\%), suivi par l'exploitation d'une plateforme d'enseignement à distance (EAD) (40\%). Un tiers des répondants confirment l'utilisation des réseaux sociaux avec leurs apprenant-es. L'utilisation des forums de discussion (19\%) et des outils de communication synchrone reste cependant très faible.

Le taux de l'utilisation de plateforme (40\%) et du forum (19\%) confirmant bien que quelle que soit la discipline, l'usage de l'Internet pour le suivi des étudiant-es après les cours est une pratique peu courante dans l'enseignement supérieur tunisien.

À ce niveau de l'analyse, nous remarquons aussi que les enseignant-es utilisant déjà un logiciel pour la présentation de leurs cours sont plus enclins à utiliser les différentes TIC pour le suivi de leurs étudiant-es.

\section{2) L'infusion pédagogique}

Dans cette partie, nous nous sommes intéressés aux 94 enseignant-es qui dispensent des cours en ligne via la plateforme Moodle de l'UVT. Ces cours sont appelés au sein de la communauté universitaire tunisienne " cours intégrés ». Ils sont dispensés en présentiel (présentiel enrichi), de façon mixte (cours hybrides) ou entièrement à distance.

Les enseignant-es chargés de ce type particulier de cours intégrés représentent 30\% des 314 enseignant-es participant à notre enquête. Afin de les distinguer des enseignant-es qui n'enrichissent pas suffisamment leurs cours en TIC, nous les appellerons : les enseignant-es de la formation intégrée « $\mathrm{FI} »$.

La répartition de ces enseignant-es $\mathrm{FI}$ en fonction de la catégorie disciplinaire du cours qu'ils et elles ont rendu accessible via la plateforme Moodle de I'UVT montre le poids des sciences et de technologie (68\%). Les cours des 1ère années licence (52\%) sont toutefois beaucoup plus accessibles via la plateforme Moodle de l'UVT que ceux des 2ème années licence 1 (22\%). En effet, la majorité des cours en ligne via les plateformes de l'UVT sont des cours «PC2i1 » et « PC2i2 » qui sont enseignés en première année licence (L1) et sont à la charge du correspondant C2i.

Le nombre de cours en ligne, déclaré par les enseignant-es, reste très limité par rapport au nombre total de cours enseignés dans ces institutions. Cela peut être expliqué par l'absence d'objectifs stratégiques au sein de ces établissements en termes de mise en ligne de cours comme le prévoit la politique nationale annoncée par le ministère de l'enseignement supérieur.

\footnotetext{
${ }^{1}$ Le diplôme Licence dure 3 ans ( L1, L2, L3 - soit un diplôme de niveau bac+3). Ce diplôme est équivalent à un Bachelor : http://parcours-Imd.salima.tn/.
} 
Nous relevons également que ce nombre reste très inférieur aux statistiques publiées par l'UVT concernant le nombre de cours en ligne accessibles via ces plateformes ou par rapport aux statistiques du programme Ingénierie de la Formation en Ligne (IFeL) de cette université. En effet, ce programme vise à accompagner les enseignant-es tunisien-es à structurer, scénariser et mettre en ligne leurs cours. Nous en déduisons que les espace de cours développés dans le cadre de ces formations restent à un état expérimental et ne sont pas exploités dans le cadre des programmes officiels. Nous pensons que ce faible taux est dû à l'absence de « modèles » ou de lignes directrices au sein des institutions universitaires pour les cours en ligne (présentiel enrichi ou hybride ou entièrement à distance) et à la « rigidité administrative », comme cela a été mentionné par un responsable. Cette conclusion confirmant bien que, quelle que soit l'institution, la mise en ligne de cours via les plateformes de l'UVT est une pratique peu courante dans l'enseignement supérieur tunisien.

Certains outils comme les ressources audio et vidéo sont très utiles aux enseignements. Ils nous semblent que la majorité des enseignant-es « FI » (ayant mis en ligne leurs cours via les plateformes de l'UVT) proposent des objets multimédias $(87,2 \%)$. Les cycles de formation IFeL "Médiatisation d'un cours en ligne » et «Conception et montage d'une capsule vidéo éducative » proposés par l'UVT a permis à la majorité des enseignant-es d'intégrer ces objets dans leurs supports de cours. II faut noter aussi que la majorité des enseignant-es répondantes estiment qu'ils et elles ont de bonnes compétences en TIC (64\%), et, par conséquent, maîtrisent les habiletés nécessaires pour enrichir leurs cours avec les ressources audio et vidéo.

De même, la majorité des répondant-es propose des ressources numériques variées intégrant des formats multimédias, des outils de communication, des activités d'apprentissage et d'évaluation, des outils de gestion et d'organisation, etc.

Afin de vérifier si les prédictions du modèle de Raby (2004) s'appliquent à notre étude, nous avons croisé les réponses des enseignant-es positionné-es dans le stade de l'exploration pédagogique avec les enseignant-es de la formation FI (stade d'infusion). La figure ci-dessous (Figure 1) montre qu'il est vraiment rare que ces derniers proposent des outils de communication et d'organisation sans avoir préalablement utilisé un logiciel de présentation pour leur cours. 
Figure 1. Tri croisé de l'utilisation d'un logiciel de présentation

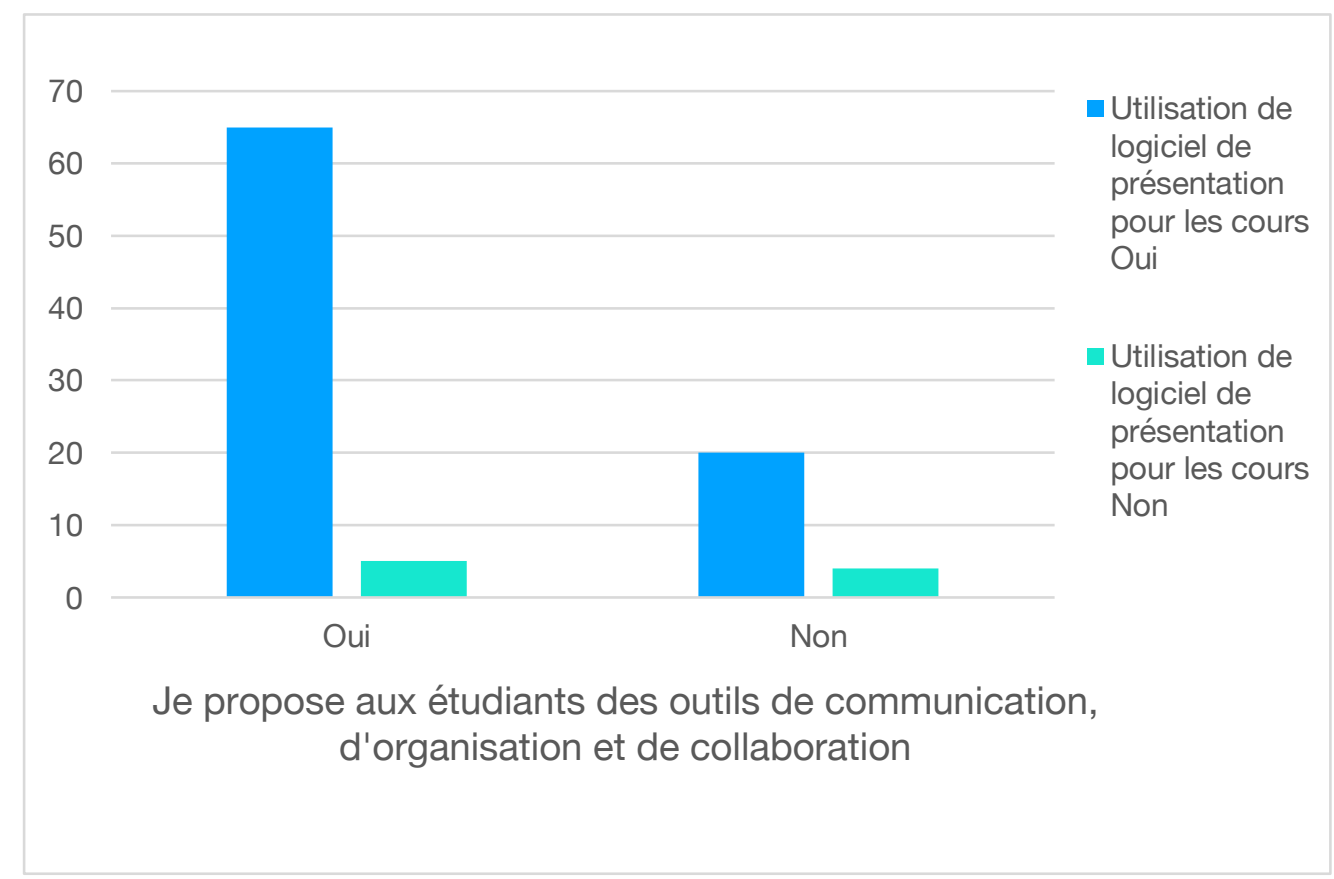

D'autres résultats réconfortent le principe selon laquelle l'infusion pédagogique des TIC est souvent précédée par une phase d'exploration. En effet, dans la figure 2 ci-après, il parait clairement que l'utilisation des différentes TIC pour le besoin du suivi des étudiant-es est nettement plus forte chez les enseignant-es qui proposent des outils d'organisation et de communication d'une manière fréquente. 


\section{Figure 2. Les outils d'organisation et le suivi des étudiant-es}

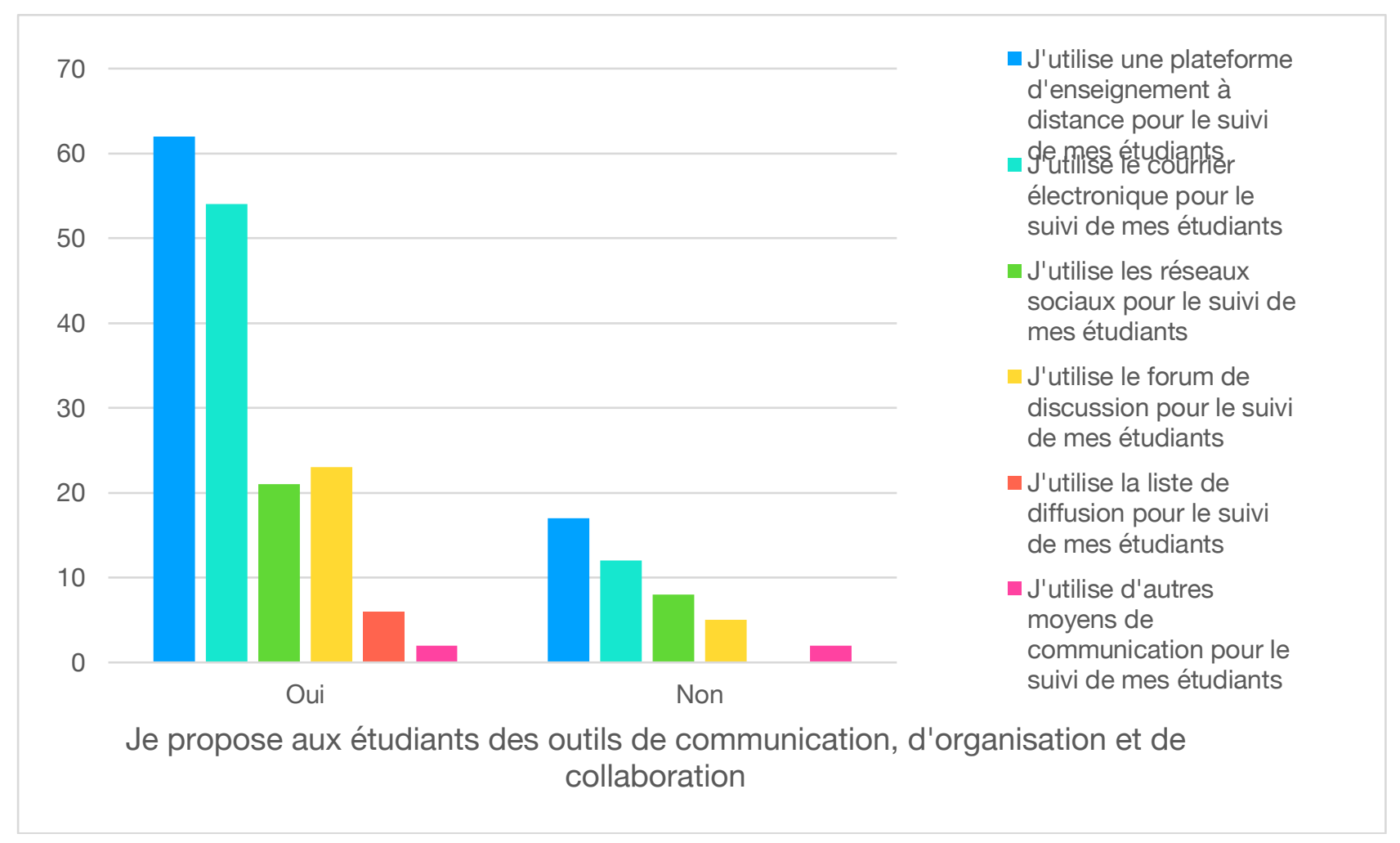

\section{3) L'appropriation pédagogique}

Pour cette partie de l'enquête, les enseignant-es pratiquant de l'appropriation pédagogique sont ceux qui proposent à leurs étudiant-es un ou plusieurs outils en ligne de production de travaux (wiki, éditeur de carte conceptuelle, blog, pad partagé, carnet de bord, etc.). L'appropriation nécessite en effet une implication plus sérieuse et fréquente des enseignantes dans la transmission et la construction des connaissances dans un cadre d'apprentissage actif. Les résultats de notre enquête montrent que les enseignant-es correspondant à ce stade avancé d'utilisation pédagogique des TIC ne sont pas très nombreux/euses : seulement 47 enseignant-es, c'est-à-dire 15\% de l'ensemble des enseignant-es interrogé-es. Le taux plus élevé de ces enseignant-es est à l'ISITC HS avec un taux de 57\%.

A ce stade de l'utilisation, nous constatons que l'outil le plus utilisé pour le suivi des étudiantes après les séances de cours n'est plus le courriel (81\% des enseignant-es) mais plutôt les plateformes d'enseignement à distance. Nous remarquons aussi que les autres outils comme les réseaux sociaux (40\%) et les liste de discussion (36\%) sont plus développés à ce stade que dans le stade de l'infusion et de l'exploration.

On constate que la majorité des enseignant-es de ce stade de l'appropriation proposent à leurs étudiant-es des outils de communication synchrone et de collaboration tels que la webconférence, le partage d'écran ou la classe virtuelle. Nous pensons que les objectifs de la majorité des cours proposés (32 cours sur les 47) pourraient viser l'appropriation de ces outils. Dans cette optique, l'utilisation poussée de ces outils collaboratifs n'est pas surprenante. 
Enfin, nous avons souhaité vérifier, à ce stade de l'utilisation, si les étudiant-es intègrent dans leurs travaux des ressources tels que des photos, des images, du son et de la vidéo. Le croisement des variables «Discipline» et "Travaux des étudiant-es » nous permet de constater que ce sont les enseignant-es informaticien-nes qui exigent ce genre de livrable des étudiant-es. Ils et elles disposaient de la valeur la plus importante avec un taux de 82\%.

Pour la grande majorité de ces enseignant-es, force est de constater que la plateforme de I'UVT est perçue comme utile et facile à utiliser. Ce qui constitue dans le modèle de l'acceptation de la technologie de Davis (1986), un facteur d'appropriation favorable à l'adoption de cette technologie par les enseignant-es. En effet, comme le montre la figure suivante, $92 \%$ des enseignant-es interrogés trouvent que la plateforme est utile, voire indispensable à la formation à laquelle ils participent.

D'autre part, 88\% des enseignant-es interrogés trouvent que l'utilisation de la plateforme de I'UVT est facile, même très facile. Pour les 12\% des enseignant-es qui trouvent difficile l'usage de cette plateforme, il convient de continuer à leur proposer un accompagnement et des formations pour les aider à s'y familiariser.

Dans la même perspective, nous avons également analysé le cadre de la formation des enseignant-es. Comme le montre la figure 3, nous avons relevé que la grande majorité, soit 86\% des enseignant-es situés dans le stade de l'appropriation pédagogique, ont poursuivi leur formation en utilisation des TIC dans le cadre du programme de formation des formateurs de l'UVT.

\section{Figure 3. Le cadre de la formation en TIC des enseignant-es de la formation intégrée}

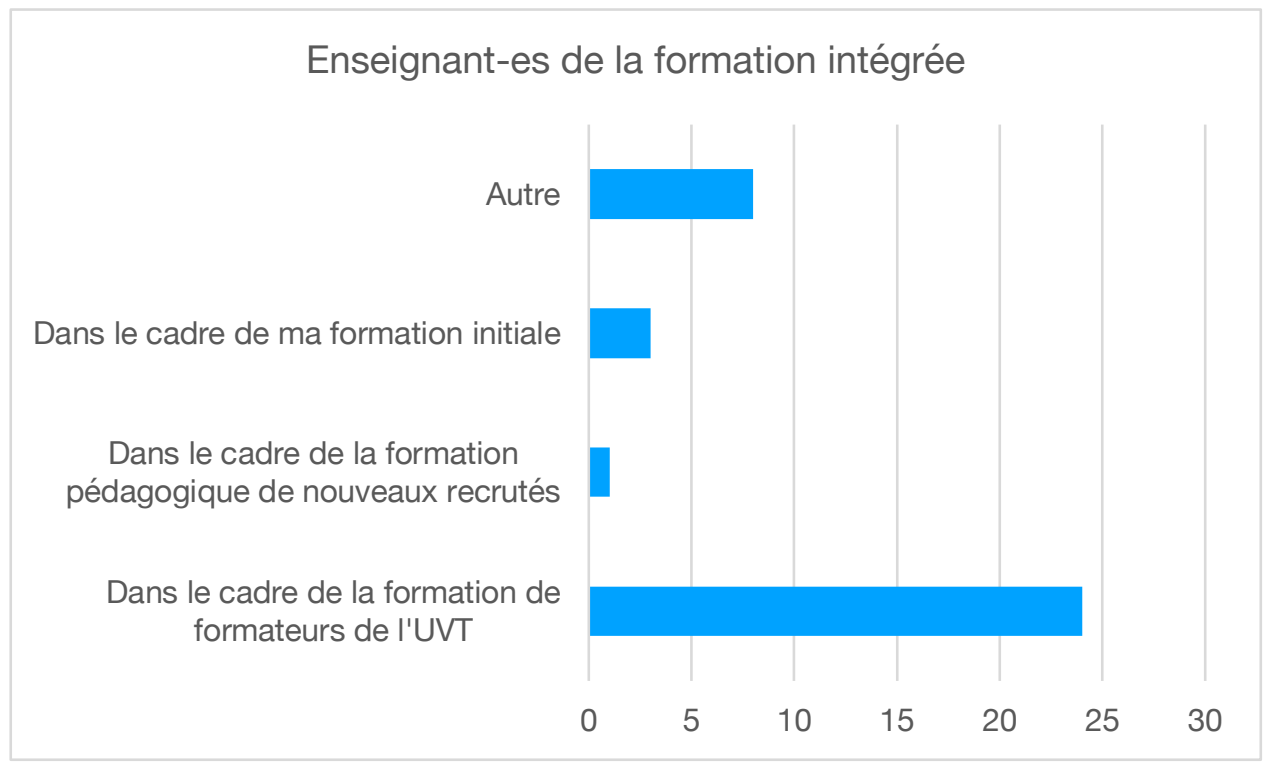




\section{CONCLUSION}

L'analyse quantitative de l'enquête destinée aux enseignant-es des cinq institutions universitaires retenues nous a permis de dévoiler une multitude de processus de mise en ligne de cours. Des expériences assez diversifiées dans le domaine de l'intégration pédagogique des TIC, au sein des établissements universitaires malgré des stratégies institutionnelles frileuses ont également été repérées.

Nous synthétisons ainsi les principaux résultats de notre analyse :

- La majorité des enseignant-es universitaires dispose de leur équipement personnel et dispose d'une connexion internet à domicile ;

- 53\% des répondant-es ont fait la démarche volontaire de se former en TIC. Près des deux tiers de ces enseignant-es déclarent avoir été formés dans le cadre des sessions de formations assurées par I'UVT. Nous soulignons également que $73,2 \%$ des enseignant-es interrogé-es ont une relation directe (correspondant-e, tuteur/trice, formateur/trice, etc.) avec I'UVT, soit un pourcentage très important. Ceci s'explique par l'approche centralisatrice de l'intégration des TIC dans l'enseignement supérieur tunisien et le rôle assigné à l'UVT dans la politique nationale ;

- 75\% des enseignant-es pensent que l'intégration pédagogique des TIC est insuffisamment développée dans leurs institutions. Ils mettent en avant l'insuffisance des équipements disponibles et la médiocrité de la qualité de la connexion internet au sein de leurs établissements. Ils ont également signalé que le soutien pédagogique n'est généralement pas assuré par l'institution. En nous appuyant sur ces résultats, nous pouvons affirmer que l'enseignement supérieur est à la traine dans l'intégration pédagogique des $\mathrm{TIC}$;

- L'analyse des réponses des enseignant-es au questionnaire nous a permis de montrer une disparité au niveau de l'investissement des universités en matière d'intégration des TIC. Cette disparité a été également notée au sein d'un même établissement et pour différentes disciplines. Nous avons donc noté l'existence d'une fracture numérique non seulement entre différentes universités, mais aussi au sein d'un même établissement de l'enseignement supérieur tunisien.

En adoptant le modèle de Raby (2004), qui présente quatre stades du processus complexe que traversent les enseignant-es en exercice lorsqu'ils et elles évoluent de la non-utilisation à I'utilisation exemplaire des TIC, l'analyse de l'enquête a permis de brosser un portrait des multiples usages des TIC par les enseignant-es tunisien-nes :

- La moitié des enseignant-es interrogé-es sont au stade de l'exploration pédagogique. En effet, ils et elles utilisent les TIC comme un support d'enseignement magistral. Ces enseignant-es proposent des supports de cours numérisés et accessibles en ligne à leurs étudiant-es. Ils et elles utilisent un logiciel de PreAO et l'internet dans les séances de cours ; 
- $15 \%$ des enseignant-es sont au stade de l'infusion pédagogique. Ils et elles offrent leurs cours via une plateforme d'enseignement à distance et utilisent des fonctions simples de cette plateforme pour le suivi et l'encadrement en ligne de leurs étudiant-es ;

- 15\% également sont au stade de l'appropriation. En effet, ils et elles utilisent une plateforme d'enseignement à distance pour assurer leurs cours et proposent des supports pédagogiques interactifs et des activités d'apprentissages innovantes aux étudiant-es. Ce type d'usage des TIC correspond au stade le plus avancé et le plus complexe du processus de l'intégration des TIC en éducation.

Aux vues de ces résultats, l'utilisation des TIC afin d'améliorer la qualité de l'apprentissage reste encore très timide. Les usages " avancés », centrés sur l'acte d'enseignement/apprentissage et associés aux activités pédagogiques qu'elles permettent de mettre en œuvre, sont attestées de façon limitée et par un nombre restreint d'enseignant-es dans les universités tunisiennes.

Nos résultats se rapprochent de ceux dont fait part la revue de la littérature scientifique en Tunisie, au niveau des usages des enseignant-es par les TIC (Benabid, 2017 ; Kaddachi \& Bouzayane, 2017 ; Kalai, 2018 ; Mehdi, 2011). Par ailleurs, nous avons noté que les enseignant-es s'investissent progressivement dans le processus de l'innovation. Pour la majorité d'entre elles et eux, la décision d'adopter une posture d'intégration des TIC est déjà dépassée et les premières activités avec les étudiant-es sont conduites et expérimentées. Nous constatons qu'ils et elles ont dépassé le stade de « décision » et s'approchent de celui de la " mise en œuvre » qui se caractérise par l'utilisation quotidienne et l'évaluation de l'innovation technologique (Rogers, 2003). Nous soulignons également qu'une minorité d'enseignant-es en sont en stade de la « confirmation ». Ainsi, ces résultats concordent avec ceux de plusieurs recherches en Occident qui ont montré que les TIC sont peu intégrées dans les pratiques pédagogiques (Raby, et al., 2011) et que le changement au niveau des pratiques pédagogiques n'est que partiellement atteint.

De façon très globale, nous remarquons que l'innovation fait son chemin dans les institutions universitaires tunisiennes et se situe au niveau de l'« implantation » selon le modèle de Graham, et al. (2013), ou aux alentours du stade intermédiaire, entre les niveaux « adaptation » et « infusion » selon le modèle de l'UNESCO (2010). Ce stade se caractérise par la présence des TIC dans l'établissement, cependant, I'utilisation des technologies centrée sur l'apprentissage à travers la réalisation de projet complexes n'est présente que dans quelques disciplines.

\section{RÉFÉRENCES}

Allouche, B. \& Belcadhi, M. (2016). L'approche de formation des formateurs adoptée à I'Université Virtuelle de Tunis. In Actes du 6e colloque international du RIFEFF : Mieux former les enseignants dans la Francophonie. Principaux enjeux actuels et futurs (pp. 163-174). 
Benabid, F. (2017). Une plateforme Moodle dans une formation hybride diplômante: étude de l'évolution des usages. Revue internationale des technologies en pédagogie universitaire, 14(2), 24-38.

Coen, P., \& Schumacher, J. (2006). Construction d'un outil pour évaluer le degré d'intégration des TIC dans l'enseignement. Revue internationale des technologies en pédagogie universitaire, 3(3), 7-17.

Davis, F. D. (1986). A technology acceptance model for empirically testing new end-user information systems: Theory and results. Management.

Graham, C. R., Woodfield, W., \& Harrison, J. B. (2013). A framework for institutional adoption and implementation of blended learning in higher education. The Internet and Higher Education, 18, 4-14. http://doi:10.1016/j.iheduc.2012.09.003

Houissa, H. R. (2009). Les pratiques hybrides de l'enseignement des TIC /C2i : Expérience des ISET en Tunisie : Cas de I'ISET-Charguia. In TICEMED: Ubiquitous Learning. https://isdm.univ-tIn.fr/PDF/isdm39/Article_Isdm_Ticemed09_Rekaya_PhD ok.pdf

Jelmam, Y. (2012). Évaluation des compétences numériques acquises suite à une formation C2I. Cas d'étudiants tunisiens. Questions vives - Recherches en éducation, 7(17), 157-170.

Kaddachi, B. (2017). Les TIC en enseignement supérieur tunisien : une intégration encore à ses premiers balbutiements. Revue internationale des technologies en pédagogie universitaire, 14(1), 57-68.

Kalai, L. (2018). Les résistances aux TIC dans le contexte tunisien: à qui la faute? In TIC et innovation pédagogique dans les universités du Maghreb (pp. 88-105).

Karsenti, T., Savoie-Zajc, L., \& Larose, F. (2001). Les futurs enseignants confrontés aux TIC: changements dans l'attitude, la motivation et les pratiques pédagogiques. Éducation et francophonie, 29, 86-124.

Khezami, S. (2016). Les institutions d'apprentissage à distance. Stratégies (politique, pédagogique et communicationnelle) et processus d'autorégulation d'apprentissage : le cas de l'Université virtuelle de Tunis. Thèse de doctorat, Université de Toulon.

Mehdi, A. (2011). Les TIC dans l'enseignement de la géographie universitaire en Tunisie: état des lieux. EpiNet, 135. https://www.epi.asso.fr/revue/articles/a1105c.htm

Moersch, C. (1995). Levels of Technology Implementation (LoTi): A Framework for Measuring Classroom Technology Use. Learning and Leading With Technology, 23, 40-42. http://doi:10.1002/ca.10103

Raby, C. (2004). Analyse du cheminement qui a mené des enseignants du primaire à developper une utilisation exemplaire des Technologies de l'Information et de la Communication (TIC) en classe. Thèse de doctorat, Université de Québec à Montréal.

Raby, C., Karsenti, T., Meunier, H., \& Villeneuve, S. (2011). Usage des TIC en pédagogie universitaire: point de vue des étudiants. Revue internationale des technologies en pédagogie universitaire, 8(3), 6-19.

Rogers, E. M. (2003). Diffusion of Innovations Theory. New York: Free Press.

Tabei, F. (2009). Approche communicationnelle du recours aux TIC dans l'enseignement supérieur tunisien: le cas du projet de l'enseignement à distance aux Instituts supérieurs des Etudes technologiques. Thèse de doctorat, Université de Grenoble.

Tabei, F. (2010, June 15). L'enseignement a distance en Tunisie : un nouveau dispositif 
universitaire en évolution. The First International Conference on e-Learning For All

[LEAFA 2010], Hammamet, Tunisie. http://pf-mh.uvt.rnu.tn/59/

Trouche, L., Emin, V., Fesselier, Y., Fontanieu, V., Goasdoué, G., Khaneboubi, M., \& Rey, O. (2014). Les recherches francophones en TICE dans le pourtour sud de la Méditerranée: Réponse à l'appel d'offres de I'IFIC (Institut de la Francophonie pour l'ingénierie de la connaissance et la formation à distance). https://hal.archives-ouvertes.fr/hal01546659/document

UNESCO. (2008). ICT Compentency Standards for Teachers. Implementation Guidelines. Paris: UNESCO.

UNESCO. (2010). Guide de mesure pour l'intégration des technologies de l'information et de la communication (TIC) en éducation. Montréal, QC : Institut de statistique de l'UNESCO.

Zghidi, S. (2010). Contribution à l'évaluation de l'intégration des TIC dans les dispositifs d'enseignement à distance : étude appliquée à l'Université Virtuelle de Tunis. Thèse de doctorat en sciences de l'information et de la communication, Université Aix-Marseille.

Open Access Publications - Bibliothèque de I'Université de Genève Creative Commons Licence 4.0

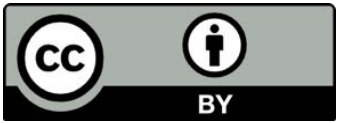

